\title{
On the Relationship Between Disbond Growth and the Release of Strain Energy
}

\author{
J.A. Pascoe ${ }^{\mathrm{a}, *}$, R.C.Alderliesten ${ }^{\mathrm{a}}$, R. Benedictus ${ }^{\mathrm{a}}$ \\ ${ }^{a}$ Structural Integrity $\& 6$ Composites Group, Faculty of Aerospace Engineering, Delft University of Technology, P.O. Box \\ 5058, 2600 GB Delft, The Netherlands
}

\begin{abstract}
Current prediction methods for growth of disbonds under fatigue loading are generally based on a correlation with either the maximum strain energy release rate (SERR) or the SERR range. This paper highlights some issues with this approach. In particular, it is argued that the maximum SERR or the SERR range alone do not give sufficient information to uniquely characterize the driving force for crack growth. Furthermore it is argued that the relationship between crack growth rate and loss of strain energy should be considered on the scale of the entire load cycle. By means of disbond growth experiments it is shown that there is indeed a very strong correlation between the crack growth rate and the strain energy lost during a fatigue cycle. Unlike methods based on the SERR, this correlation is not affected by the R-ratio. Based on the found correlation a possible basis for a new approach to disbond growth prediction is suggested.
\end{abstract}

Keywords: Adhesive Bonding, Crack Growth, Fatigue, Fracture Mechanics

\section{Note to the reader}

This is the accepted author manuscript version of an article submitted to Engineering Fracture Mechanics. It does not include type-setting and copy-editing performed by the publisher. The version of record of this article is:

J.A. Pascoe, R.C. Alderliesten, R. Benedictus, (2015) 'On the relationship between disbond growth and the release of strain energy', Engineering Fracture Mechanics, 133, 1-13,

DOI: http://dx.doi.org/10.1016/j.engfracmech.2014.10.027

\section{Introduction}

A lack of understanding of the fatigue disbonding behaviour of adhesive bonds is one of the major obstacles preventing their wide-spread application as a primary joining method in the aerospace industry. Until the growth of disbonds under fatigue loading can be accurately predicted, adhesive bonding's promise of more efficient (i.e. lighter) structural joints will go unfulfilled. This challenge has of course been recognised for quite some time, and over the past four decades many researchers have investigated fatigue disbond growth (FDG). However, as the present authors have discussed elsewhere [1], these investigations have mainly resulted in a large collection of empirical correlations of most likely limited validity. This paper presents a consideration based on the energy balance considered on a per-cycle $(\mathrm{d} / \mathrm{d} N)$, rather than an instantaneous, per-crack-extension, $(\mathrm{d} / \mathrm{d} a)$ basis. The discussion will be limited to FDG by means of growth

\footnotetext{
${ }^{*}$ Corresponding author

Email address: j.a.pascoe@tudelft.nl (J.A. Pascoe)
} 
of cracks in the adhesive layer (i.e. cohesive failure of the adhesive) and thus the terms disbond and crack may be considered interchangeable throughout this paper.

This paper will start with a historical review of the development of current approaches to the prediction of crack growth rate. Several issues with these methods will be discussed, leading to the hypothesis that crack growth rate is correlated to the total amount of energy lost during a fatigue cycle, and not to the strain energy release rate (SERR). The next section will describe the experiments performed to test this hypothesis, followed by a discussion of the results of these tests. Finally the applicability of the findings to the prediction of FDG will be considered.

\section{Critical analysis of the development of current approaches}

This section provides a review of the development of the current approach to understanding FDG and then highlights and discusses some shortcomings inherent in this approach.

\subsection{Historical review}

The vast majority -if not all- of the current methods for predicting FDG, even those based on finite element techniques such as the cohesive zone models or XFEM, describe the crack growth behaviour with an expression from the realm of linear elastic fracture mechanics (LEFM) [1]. The basis of these expressions was formed by the work of Griffith [2], who proposed that to create or propagate a crack, one has to supply a quantity of energy equal to the surface energy of the material multiplied by the area of the new fracture surfaces.

The theory as proposed by Griffith was only applicable to perfectly brittle materials. Orowan extended this theory to ductile materials, by adding the energy dissipated by plastic deformation in the vicinity of the crack tip to the consumption side of the energy balance [3, 4]. This was also independently proposed by Irwin [5].

The consumption side of the energy balance for crack growth thus being determined, attention shifted to the supply side. Irwin and Kies [6] pointed out that Griffith had already shown that growth of a crack would cause a release of strain energy. Based on this finding Irwin and Kies argued [5-7] that under fixed grip conditions the energy required for crack growth must be supplied by the strain field surrounding the crack. Thus, they argued, a crack can grow only if the rate of release of strain energy per unit area of crack growth $(d U / d A)$ exceeds the energy required per unit area of crack growth $(d W / d A)$. This is now more commonly stated as:

$$
G \geq G_{\mathrm{C}}
$$

where $G$ is the SERR. Note that this is a stability criterion. If a hypothetical crack extention would release more energy than required for crack growth, then the crack growth is self-sustaining and thus unstable cracking will occur. Equation 1 determines whether crack growth can occur without outside energy input, but gives no information on what the rate of crack growth will be.

Irwin [8] extended the SERR concept further by analysing the Westergaard stress functions [9]. He argued that when the solutions given in [9] are converted to use polar coordinates, the resulting stress is a function of a stress intensity factor (SIF) and a series of trigonometric functions. By equating the work done to open the crack to the strain energy release rate, Irwin demonstrated the equivalence of the SERR and the SIF, which is now usually written as [10, 11]:

$$
G=\frac{K^{2}}{E^{\prime}}
$$

where $K$ is the SIF and $E^{\prime}$ is a stiffness that depends on the stress/strain state. In the case of plane stress $E^{\prime}=E$, and for plane strain $E^{\prime}=E /\left(1-\nu^{2}\right)[10]$, where $E$ is Young's modulus and $\nu$ is Poisson's ratio. Note that strictly speaking this equation only holds if $K_{a}=K_{a+\Delta a}$, i.e. if the growth of the crack does not affect the value of the SIF. Also note that while $K$ always is a real quantity, as there is always a stress field with a certain intensity, $G$ is a virtual quantity. It is the amount of energy that would be released per unit 


\begin{tabular}{|c|c|c|c|}
\hline \multicolumn{4}{|c|}{ Nomenclature } \\
\hline$A$ & Disbond area $\left(\mathrm{mm}^{2}\right)$ & $P$ & Force $(\mathrm{N})$ \\
\hline$a$ & Disbond length $(\mathrm{mm})$ & $R$ & Ratio of minimum to maximum force \\
\hline$C$ & Parameter in the Paris relation & $U$ & Strain energy (mJ) \\
\hline$d$ & Displacement (mm) & $W$ & Work of fracture $(\mathrm{mJ})$ \\
\hline$E$ & Young's modulus (MPa) & $w$ & Specimen width $(\mathrm{mm})$ \\
\hline$E_{k}$ & Kinetic energy $(\mathrm{mJ})$ & \multicolumn{2}{|c|}{ Greek Symbols } \\
\hline$F$ & Work (mJ) & & Poisson's ratio \\
\hline$G$ & Strain energy release rate $(\mathrm{N} / \mathrm{mm})$ & \multicolumn{2}{|c|}{ Subscripts } \\
\hline$G^{*}$ & $\begin{array}{l}\text { Average strain energy release rate } \\
(\mathrm{N} / \mathrm{mm})\end{array}$ & $\mathrm{c}$ & Critical \\
\hline$I$ & Moment of inertia $\left(\mathrm{mm}^{4}\right)$ & cyc & Cyclical \\
\hline$K$ & Stress intensity factor $(\mathrm{MPa} \sqrt{\mathrm{mm}})$ & $\min$ & Minimum \\
\hline$N$ & Number of cycles & $\max$ & Maximum \\
\hline$n$ & Parameter in the Paris relation & mono & Monotonic \\
\hline$n$ & Compliance calibration parameter & tot & Total \\
\hline
\end{tabular}

of crack extension if there is a crack extension. If there is no crack extension there will also be no release of energy, although a $G$ value can still be computed. With equation 2 Irwin transformed a virtual release of energy into a measure for the intensity of the stress field surrounding a crack tip.

All the work discussed so far was done for cracks under quasi-static loading. However Paris and coworkers argued that under fatigue loading, the crack growth behaviour is also governed by the stress field surrounding the crack tip, and that $K$ is a good measure for the intensity of that stress field [12]. This lead to the proposing of the famous Paris relation $[13,14]$ :

$$
\frac{d a}{d N}=C \Delta K^{n}
$$

where $C$ and $n$ are determined by curve fitting. Although it is now common to consider both $C$ and $n$ as material parameters, it is worth noting that Paris explicitly considered $C$ to be a function of the mean stress (or one could state equivalently; R-ratio) [13].

Paris and co-workers investigated crack growth in metals. Their work was adapted to interface crack growth in a metal-composite laminate by Roderick et al. [15] and to crack growth in an adhesive bond by Mostovoy and Ripling [16]. Due to the difficulty of computing $K$ for these cases, Equation 2 was used to convert the Paris relation to a relationship based on $G$. Thus Roderick et al. proposed the relationship [15]:

$$
\frac{d a}{d N}=C G_{\max }^{n}
$$

and Mostovoy and Ripling proposed [16]:

$$
\frac{d a}{d N}=C \Delta G^{n}
$$

with $\Delta G=G_{\max }-G_{\min }$, and $C$ and $n$ again empirically determined by curve fitting.

Starting from these two equations many models have been proposed to predict the disbond growth rate. However upon examination it becomes clear that they are nearly all variations on the basic theme of a power-law relationship between disbond growth rate and either $G_{\max }$ or $\Delta G$ [1]. 


\subsection{Shortcomings in the current approaches}

One issue that has received much attention in the work that followed that of Roderick et al. and Mostovoy and Ripling, is the so-called 'R-ratio effect', i.e. the effect of the ratio of minimum to maximum load. This is somewhat of a misnomer. The term R-ratio effect implies that there exists a fundamental relationship between the FDG and e.g. $G_{\max }$ or $\Delta G$, and that this fundamental relationship is modified by the R-ratio. One can easily see that it is in fact something else that is the case however. One parameter (i.e. either $G_{\max }$ or $\Delta G$ alone) is insufficient to uniquely describe a load cycle. The FDG rate depends on the entire load cycle. Thus the so-called R-ratio effect is really only a reflection of the inadequacy of using only $G_{\max }$ or $\Delta G$ to describe the load cycle. To uniquely describe a load cycle, one requires two parameters, e.g. both $G_{\max }$ and R, or $\Delta G$ and $G_{\text {mean }}$. This can be done by explicitly including the R-ratio in the equations, as done e.g. in [17-21] or by formulating equations that depend on both $G_{\max }$ and $\Delta G$, see e.g. [22-30].

There is a more fundamental point here though. In the models mentioned above, the used approach was chosen to account for a perceived 'R-ratio effect', and usually based upon some empirical correlation, rather than an a priori consideration of the physics of the problem. Let us therefore now consider the underlying physics by returning to the energy balance proposed by Irwin and Kies [6, 7]. They stated that for fixed grip conditions and neutral stability of the crack growth, the SERR equals the energy consumed by crack growth. This can be generalised to non-fixed grip conditions as [11]:

$$
\frac{d F}{d A}-\frac{d W}{d A}=\frac{d U}{d A}+\frac{d E_{k}}{d A}
$$

where $F$ is the work done by the forces on the boundary of the specimen, $U$ is the internal strain energy and $E_{k}$ is the kinetic energy, which can usually be neglected for the class of problems being considered here. Assuming $d E_{k} / d A=0$ and rearranging the terms to a form more closely matching the formulation proposed by Irwin and Kies gives:

$$
G_{\mathrm{c}}=\frac{d W}{d A}=\frac{d F}{d A}-\frac{d U}{d A}=G
$$

This equation implies $G$ represents the energy supplied to the crack, enabling growth. Indeed, Irwin suggested that $G$ could be regarded as the driving force for crack growth [8]. However, $G$ is the energy that is released when the internal constraints in the specimen are removed by crack growth. In other words the energy release described by $G$ is a consequence of crack growth. Thus, in the authors' opinion, it is preferable not to refer to $G$ or $\Delta G$ as the crack driving force. In the case of a crack under quasi-static loading conditions, where the equality of $G$ and $G_{\mathrm{c}}$ represents a stability criterion, this distinction is not so important. If one increment of crack growth releases an amount of energy equal to, or greater than, the energy required for that crack growth (i.e. $G \geq G_{\mathrm{c}}$ ) then the crack growth is clearly self-sustaining without external energy input, i.e. unstable.

In fatigue however, the case becomes very different. Crack growth in fatigue occurs for $G$ values significantly below $G_{\mathrm{c}}$, or at least below the value of $G_{\mathrm{c}}$ found in quasi-static tests. Additionally, FDG involves stable crack growth: a test machine can be paused at any point during the load cycle, maintaining the load on the specimen, and the crack will not grow further. This holds true, even though simply pausing the test machine does not change the instantaneous value of $G$. Thus one can conclude that equation 7 does not tell the complete story in fatigue.

What precisely happens at each instantaneous moment during the fatigue cycle is at yet unclear. However if one zooms out to the time-scale of one fatigue cycle, a general description can be given. Griffith's concept of crack growth requiring energy must still hold true. Thus, assuming for convenience unit width, one can state that during one cycle there will be an amount of crack growth $\Delta a=d a / d N$. This crack growth will require an amount of energy equal to $W \Delta a$, where $W$ is the amount of energy required per unit of crack growth. $W$ includes both the energy required for the severing of bonds to allow crack growth, and any additional energy dissipating mechanisms in the vicinity of the crack tip (e.g. plastic deformation). If one considers a fatigue test, during the loading half of the fatigue cycle the test machine will supply energy to the specimen, which is stored in the form of strain energy. In this paper this energy is referred to as $U_{\text {cyc }}$, as defined in figure 1. During the unloading half of the fatigue cycle, this energy will be returned. The amount 
of energy that is returned is not necessarily equal to the amount of energy supplied, as dissipation of energy may occur due to processes such as crack growth and plastic deformation.

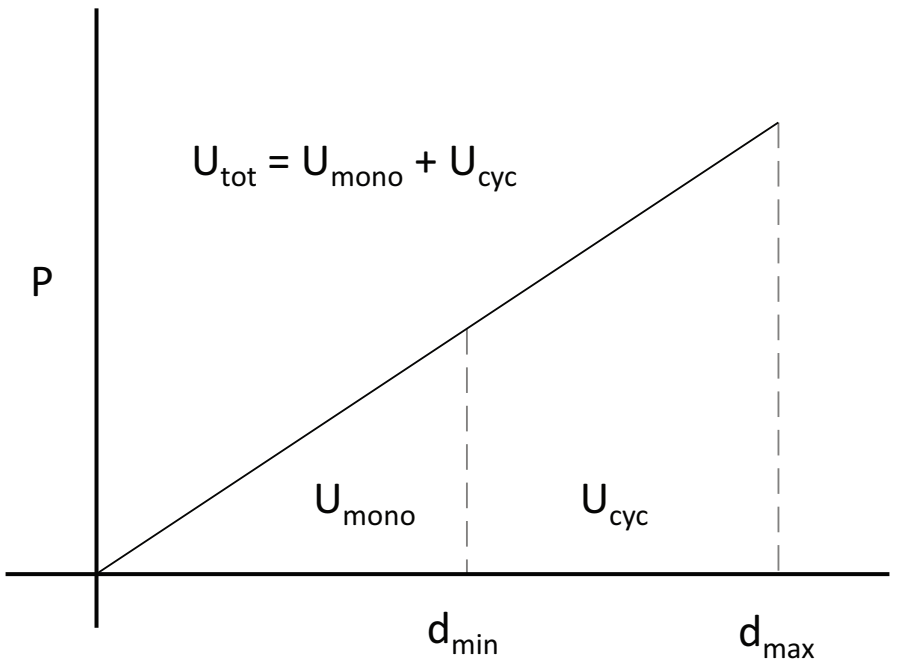

Figure 1: Schematic force $(P)$ - displacement $(d)$ diagram, showing the definition of $U_{\text {mono }}, U_{\text {cyc }}$ and $U_{\text {tot }}$.

If the test is being conducted under displacement control, this means that the amount of energy contained in the cycle will steadily decrease, i.e. $d U_{\text {cyc }} / d N<0$; the released strain energy will not be replaced by the test machine. Under load control the situation becomes more complicated, as the amount of cyclic energy will in principle increase as the crack grows. This is because crack growth reduces the stiffness of the specimen and thus increases the maximum displacement attained during the load cycle. However it is in this case still possible to determine the amount of strain energy released due to the loss of stiffness of the specimen. This will be equal to the difference in strain energy between two cycles, minus the work done as a result of the additional displacement.

As discussed above, equation 1 most likely does not hold during FDG. However based on Griffith's energy principle, at the scale of one fatigue cycle, it is hypothesised that the amount of energy released during the total load cycle must be related to the amount of energy consumed for crack growth and thereby to the crack growth rate. A series of fatigue tests was conducted to test this hypothesis.

\section{Test Set-up}

This section will first discuss the specimens used in the fatigue tests and then discuss the actual test set-ups. The specimens and the displacement controlled tests were already discussed in [31]. As a matter of convenience to the reader this information is repeated here. Further details on the specimens, including more detailed measurements, can be found in the publicly available dataset [32].

\subsection{Description of the specimens}

The specimens used were double cantilever beam (DCB) specimens, consisting of two 6 mm thick Al-2024 beams bonded together with FM94 epoxy adhesive. The nominal width of the specimens was $25 \mathrm{~mm}$ and the nominal length was $300 \mathrm{~mm}$. The post-curing thickness of the adhesive layer was approximately 0.07 $\mathrm{mm}$. Prior to bonding Teflon tape was applied to the surface of both beams over a length of $65 \mathrm{~mm}$ to prevent adhesion. As crack length is measured from the line of load application, in accordance with the ASTM D5528-01 standard [33], this resulted in a $50 \mathrm{~mm}$ pre-crack. The aluminium was bonded in the form of two plates. After bonding the joined plates were cut and milled into strips of the required dimensions. 
The aluminium was pre-treated with chromic acid anodisation and then immediately bonded. Curing of the adhesive was done in an autoclave at $120{ }^{\circ} \mathrm{C}$ and 6 bar, with a 2 hour hold time and a heating and cooling rate of $2{ }^{\circ} \mathrm{C} / \mathrm{min}$, all in accordance with the manufacturer's instructions.

One side of the specimen was coated with thinned typewriter correction fluid (in essence, a white paint) in order to enhance visibility of the crack.

\subsection{Test procedure}

Tests were performed on 4 specimens. Due to the length of the specimens several independent tests were performed on the same specimen in a number of cases. In total there were valid results for 5 crack growth experiments conducted under displacement control and 2 experiments conducted under load control. There were also several displacement controlled experiments in which no crack growth was detected and one experiment in which the load measurements were found to have been incorrectly calibrated. The test matrix for the displacement controlled tests can be found in table 1 and the test matrix for the load controlled tests is contained in table 2 . All tests were performed at $5 \mathrm{~Hz}$.

Table 1: Test matrix for the displacement controlled tests indicating the applied minimum and maximum displacement. Two independent experiments were performed on specimen B-002. Specimen codes match those of the dataset [32]. The listed R ratios are the mean values of $P_{\min } / P_{\max }$ achieved during the test. As the extrapolated P-d curve did not pass through the origin, this does not equal $d_{\min } / d_{\max }$. The standard deviations of the R-values are shown in parentheses. This table has also been published in [31] (without the standard deviation of the R-values).

\begin{tabular}{llll}
\hline Specimen \& Crack & $d_{\max }(\mathrm{mm})$ & $d_{\min }(\mathrm{mm})$ & $\mathrm{R}(-)$ and standard deviation \\
\hline C-001-I & 2.85 & 0.95 & $0.29(0.0047)$ \\
C-002-D & 2.85 & 1.90 & $0.61(0.0102)$ \\
B-001-II & 7.50 & 0.75 & $0.036(0.0060)$ \\
B-002-I & 3.16 & 2.78 & $0.86(0.0015)$ \\
B-002-II & 3.79 & 2.82 & $0.61(0.0145)$ \\
\hline
\end{tabular}

Table 2: Test matrix for the force controlled tests indicating the applied minimum and maximum force. Specimen codes match those of the dataset [32].

\begin{tabular}{llll}
\hline Specimen \& Crack & $P_{\max }(\mathrm{N})$ & $P_{\min }(\mathrm{N})$ & $\mathrm{R}(-)$ \\
\hline C-002-F1 & 300 & 50 & 0.17 \\
C-002-F2 & 200 & 50 & 0.25 \\
\hline
\end{tabular}

Tests were conducted on an MTS $10 \mathrm{kN}$ hydraulic fatigue testing machine. Prior to each fatigue test the specimens were loaded quasi-statically until onset of crack growth was seen visually, in order to generate a starter crack. Displacement and load at maximum and minimum displacement were recorded by the test machine itself at intervals of 100 or 1000 cycles, selected depending on the speed of crack growth. Crack growth was monitored by a camera aimed at the side of the specimen. Pictures were taken at the chosen recording intervals while the test machine was held at maximum displacement or load, as appropriate. A typical image is shown in figure 2. These pictures were then analysed with a simple image processing algorithm to automatically determine the crack length. Two specimens (B-002 and C-001) were completely separated using a Zwick $20 \mathrm{kN}$ hydraulic tensile test machine after the fatigue cycling had been completed to allow examination of the fracture surfaces.

\section{Data Analysis}

As mentioned above, the data gathered during the test were displacement, load, crack length and cycle number. These raw data, as well as derived values, are publicly available via the 3TU data centre [32, 34]. From the data gathered during the test, the crack growth rate was determined by taking the derivative of 


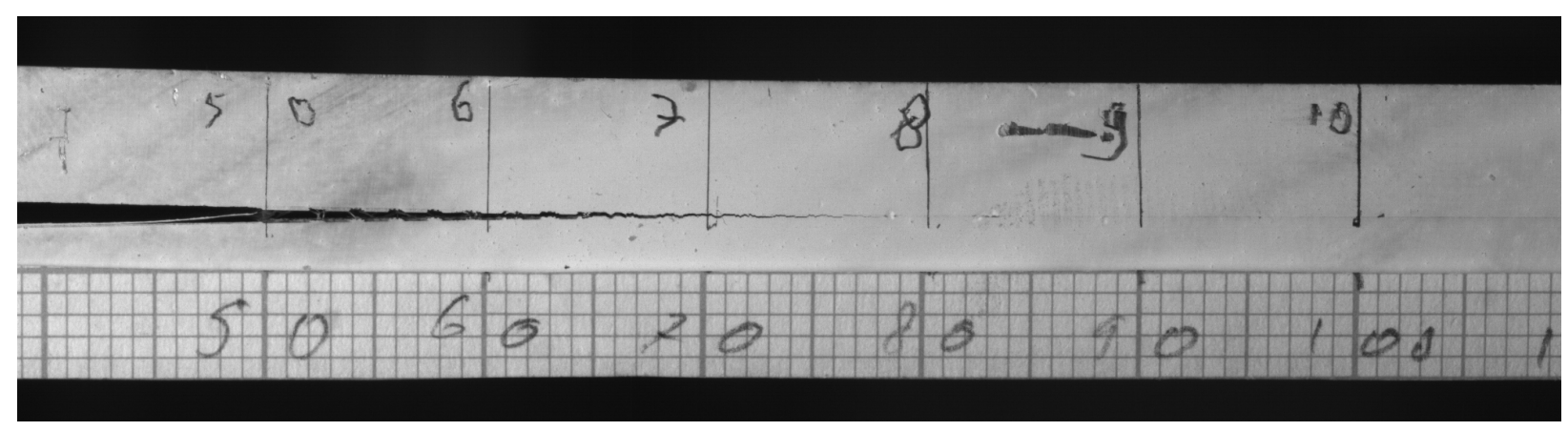

Figure 2: Crack length image captured by the camera during the test. The graph paper is graduated in $1 \mathrm{~mm}$ increments.

a curve fit through the $a$ versus $N$ data points. The SERR was computed according to the compliance calibration method given in ASTM D5528-01 [33], i.e:

$$
G=\frac{n P d}{2 w a}
$$

where $w$ is the width of the specimen, and $n$ is a compliance calibration parameter, equal to the slope of the line of $\log (C)$ versus $\log (a)$. This value was determined separately for each specimen.

The strain energy was determined by assuming the $P$ - $d$ behaviour to be linear between $\left(d_{\min }, P_{\min }\right)$ and $\left(d_{\max }, P_{\max }\right)$. The validity of this assumption will be discussed later in this section. The assumed behaviour was extrapolated to find the intersection between the $P$ - $d$ curve and the $d$-axis, as the $P$ - $d$ curve did not pass exactly through the origin. The strain energy was calculated as the area under the $P$ - $d$ curve. The calculated strain energy was then plotted against the number of cycles (see figure 3). A power-law curve could be fit through each set of measurement data. The equation for each curve was then differentiated to give the cyclic and total energy loss $d U_{\text {cyc }} / d N$, and $d U_{\text {tot }} / d N$ as a function of cycle number.

For the force controlled experiments the calculation was somewhat more involved, as under force control the strain energy will increase due to the increase in displacement. Thus the release of strain energy is equal to the difference in strain energy, minus the work done due to the increase in displacement, or algebraically:

$$
\frac{d U}{d N}=\frac{0.5 P\left(d_{N+\Delta N}-d_{N}\right)-P\left(d_{N+\Delta N}-d_{N}\right)}{\Delta N}=\frac{0.5 P\left(d_{N}-d_{N+\Delta N}\right)}{\Delta N}
$$

where $d_{N}$ is the displacement at cycle number $N, d_{N+\Delta N}$ is the displacement at cycle number $N+\Delta N$ and $\Delta N$ is the number of cycles between two measurement points. This calculation was performed for each data point. Subsequently a curve was fit through the collection of $d U / d N$ versus $N$ points in order to compensate for scatter in the measurement data.

The fitting parameters and root mean square error (RMSE) for the cyclic strain energy for the displacement controlled specimens are shown in table 3. For the force controlled specimens only the derivative of the cyclic strain energy with respect to the number of cycles was calculated, this is shown in table 4 .

Table 3: Fit parameters and resulting root mean square error (RMSE) for the cyclic strain energy as a function of cycle number for the displacement controlled specimens. All fits follow the model $U_{\mathrm{cyc}}=a N^{b}$.

\begin{tabular}{llll}
\hline Specimen \& Crack & $\mathrm{a}$ & $\mathrm{b}$ & RMSE (mJ) \\
\hline C-001-I & 1528 & -0.1519 & 1.669 \\
C-002-D & 920.7 & -0.1159 & 1.324 \\
B-001-II & 1858 & -0.136 & 2.933 \\
B-002-I & 345 & -0.061 & 9.534 \\
B-002-II & 492.1 & -0.1133 & 0.7828 \\
\hline
\end{tabular}


Table 4: Fit parameters and resulting root mean square error (RMSE) for minus 1 times the derivative of the cyclic strain energy as a function of cycle number for the force controlled specimens. C-002-F 1 was fit as $d U_{\text {cyc }} / d N=a e^{b}+c e^{d}$ and C-002-F2 was fit as $d U_{\text {cyc }} / d N=a N^{b}+c$

\begin{tabular}{llllll}
\hline Specimen \& Crack & $\mathrm{a}$ & $\mathrm{b}$ & $\mathrm{c}$ & $\mathrm{d}$ & $\mathrm{RMSE}(\mathrm{mJ})$ \\
\hline C-002-F1 & 0.007961 & 0.0003528 & $9.867 \cdot 10^{-15}$ & 0.004087 & 0.07881 \\
C-002-F2 & $1.08 \cdot 10^{-14}$ & 3.406 & 0.02765 & - & 0.0472 \\
\hline
\end{tabular}

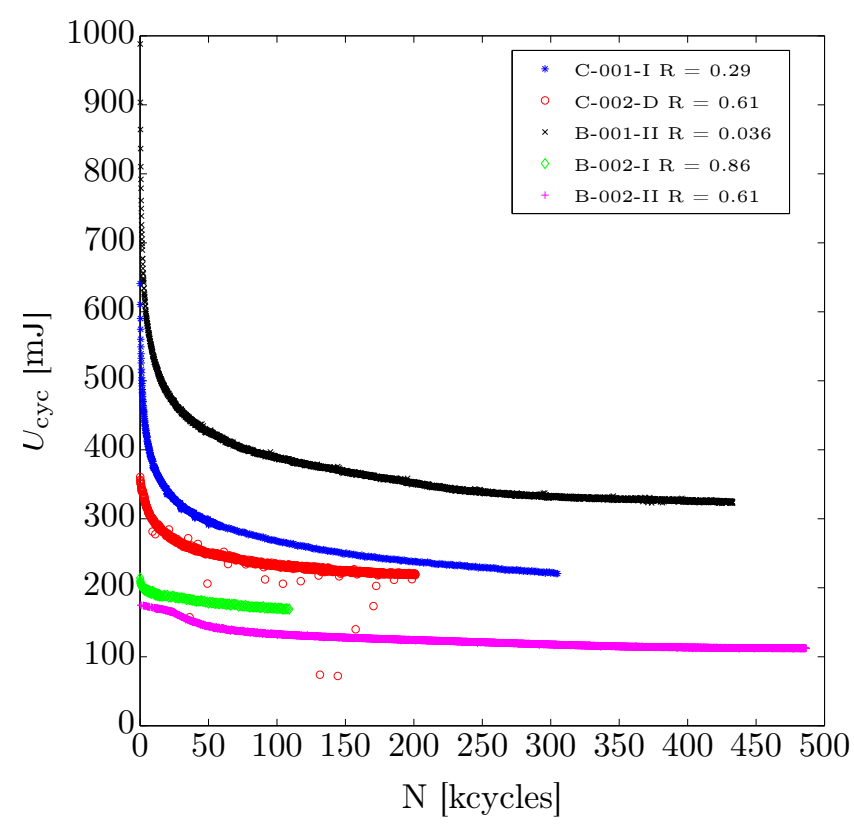

Figure 3: Cyclic strain energy versus number of cycles

\subsection{Consequences of assuming linear load-displacement behaviour}

It should be noted that assuming linear force-displacement behaviour introduces some errors in the calculated value of $U$. For the calculation it was assumed that the load-displacement curve is linear between $d_{\min }$ and $d_{\max }$. In reality however crack growth during the load cycle will cause the stiffness of specimen to diminish during the load cycle and the $P-d$ behaviour will follow a curved path, as illustrated in figure 4 . As a consequence, assuming linear behaviour will cause the calculated value of $U_{\text {cyc }}$ to be lower than the actual

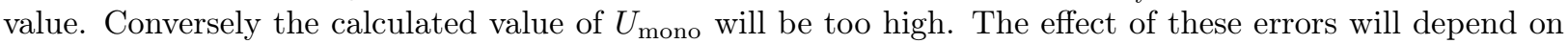
the R-ratio. A higher R-ratio, while holding $d_{\max }$ constant, will cause a larger error in the monotonic energy and a smaller error in the cyclic energy. However the size of the errors will also be affected by the absolute displacement values and by the magnitude of the crack growth.

A (somewhat crude) estimate of the error can be found as follows: From beam theory the derivative of compliance with respect to crack length can be found as

$$
\frac{d C}{d a}=\frac{2 a^{2}}{E I}
$$

where $I$ is the moment of inertia. Thus for a given crack length and corresponding crack growth rate one can estimate what the compliance would have been if crack growth was absent as:

$$
C_{\text {nocrack }}=C_{\text {measured }}+\frac{d C}{d a} \cdot \frac{d a}{d N}
$$


With this compliance value one can estimate the maximum load if there had been no crack growth. Then the strain energy for the hypothetical uncracked condition can be calculated. The difference between the strain energy for the hypothetical uncracked condition and the measured cracked condition (area ABC in figure 4) is then a first estimate of the error caused by the assumption of linearity of the load-displacement curve.

While the area between the real behaviour and the assumed behaviour for $d>d_{\min }$ (i.e. the area enclosed by the path ABA) represents a release of energy that is not accounted for, it should be noted that the area AEF represents an error in the absolute value of $U_{\text {mono, }}$, but was found not to affect the value of the derivative $d U_{\text {mono }} / d N$. Thus the magnitude of the error in the released strain energy was estimated based only on area $\mathrm{ABC}$.

A careful examination of figure 4 shows using area ABC will overestimate the magnitude of the error, especially for large values of $d_{\max }-d_{\min }$ and if crack growth already starts at low values of $d$. Thus as a first estimate of the error a value of half the difference between the uncracked and cracked strain energies was used. The magnitude of this error (i.e. half of area ABC divided by the calculated value of $d U_{\text {cyc }} / d N$ ) ranges from $4.3 \%$ for $\mathrm{B}-002-\mathrm{I}(\mathrm{R}=0.86)$ to $40 \%$ for $\mathrm{B}-001-\mathrm{II}(\mathrm{R}=0.036)$. It is emphasised that this is a rough approximation, and that it likely overestimates the magnitude of the error, especially for low R-ratios.
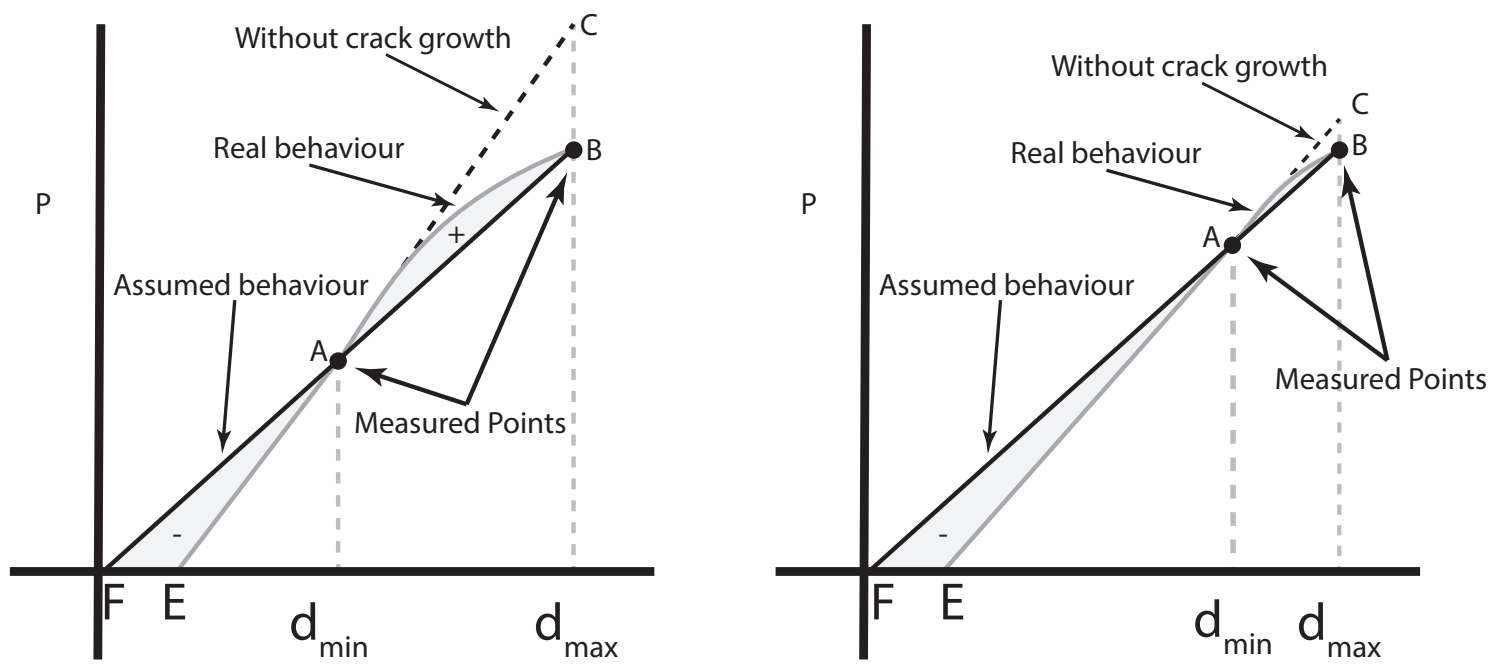

Figure 4: Errors in the calculated strain energy due the difference between the assumed behaviour and the actual behaviour. The cyclic energy is underestimated (the shaded area marked ' + ') and the monotonic energy is overestimated (the shaded area marked '-'). The left panel shows the situation for a low R-ratio and the right panel shows a high R-ratio. The area of the triangle $\mathrm{ABC}$ is used to estimate the size of the error.

\section{Results and discussion}

As mentioned in section 3.2 seven experiments resulted in valid results. The displacement controlled tests lasted between 100 and 500 kcycles and were stopped when sufficient data was deemed to have been 
collected. The force controlled tests lasted approximately 8 kcycles and were stopped when the test machine reached pre-determined displacement limits.

\subsection{Fractography}

Examination of the fracture surfaces for the separated specimens showed the presence of adhesive residue on both surfaces, indicating disbonding occurred by cohesive fracture of the adhesive. Figure 5 shows a typical example. There was a distinct colour difference visible between the area affected by quasi-static crack growth and the area affected by fatigue crack growth. This showed that the crack front was curved, but that the distance from the load line to the crack front was roughly equal on both sides of the specimen. The crack length in the centre of the specimen was longer than at the edges. As the camera used for recording the crack length was aimed at the side of the specimen, all the crack lengths mentioned in this paper were measured at the edge of the specimen. In figure 5 imprints left by the adhesive carrier fibres can be seen. It has been shown that the presence of such a carrier does not affect the crack growth rate under mode I loading [35]. Figure 6 shows a side view of the lower half of one of the cracks. As can be seen in the figure, the distance between the crack tip and the adherends varied, but the crack remained within the adhesive itself, rather than running along the adhesive-adherend interface.

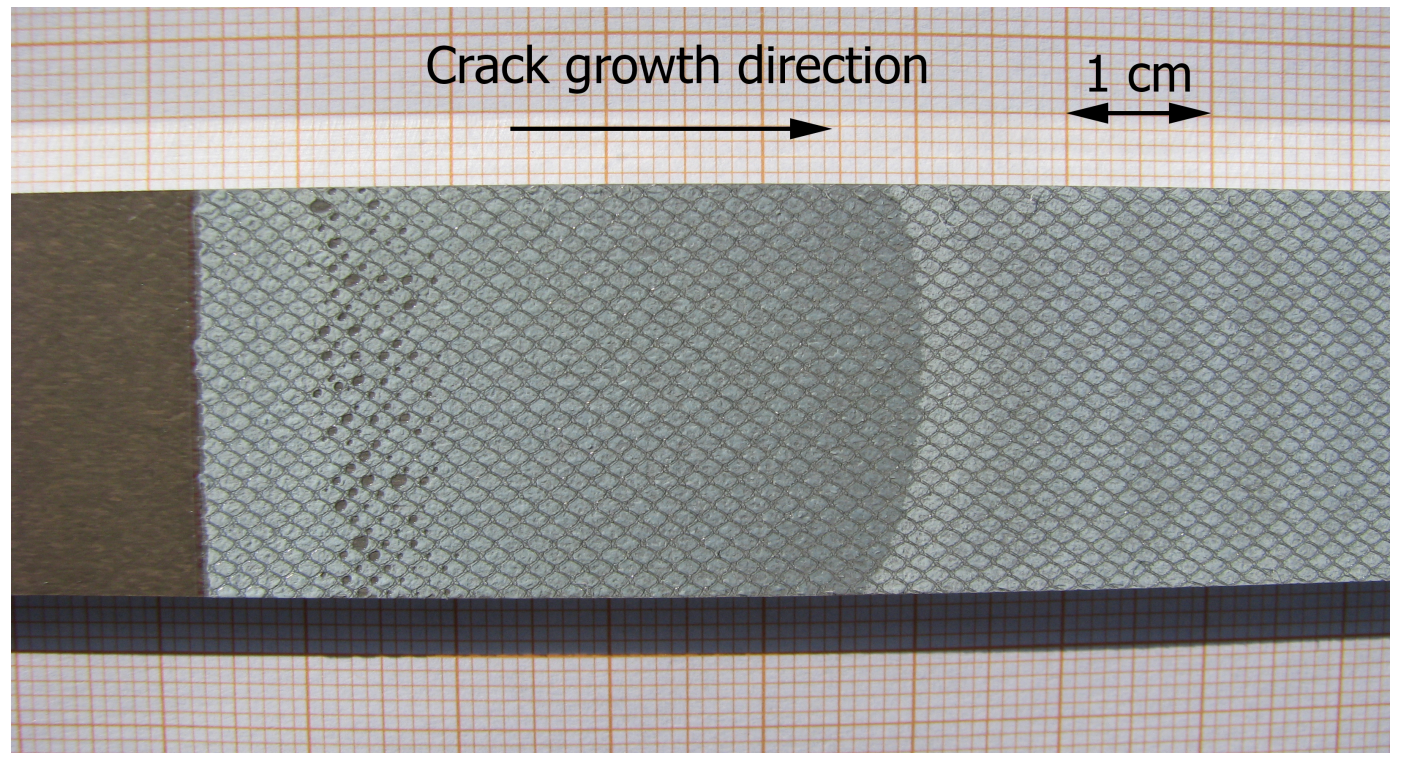

Figure 5: View of the crack surface of specimen C-001.

\subsection{Crack growth rate compared to strain energy release}

Figure 7 shows the traditional correlations between the crack growth rate and the maximum SERR and the SERR range. The SERR range was defined as $\Delta \sqrt{G}=\left(\sqrt{G_{\max }}-\sqrt{G_{\min }}\right)^{2}$, as this preserves the similarity of stress cycles implied by the use of $\Delta K$ proposed by Paris [36]. There is a clear R-ratio dependence visible in the graphs, as expected. For experiment C-002-F1 there appears to be a threshold behaviour, but it important to note that due to the self-accelerating nature of crack growth in a forcecontrolled DCB experiment, the growth rate could not be very accurately determined for low growth rates.

Figure 8 shows the correlation between the crack growth rate (in terms of additional crack surface per cycle, assuming a straight crack front) and the strain energy released during each fatigue cycle. Both the correlation between crack growth rate and loss of cyclic energy $\left(d U_{\text {cyc }} / d N\right)$ and between crack growth rate and loss of total energy $\left(d U_{\text {tot }} / d N\right)$ is shown. Again experiment C-002-F1 appears to show threshold behaviour; as before it is thought this is most likely an artefact of the measurement and data analysis techniques. The correlation between crack growth rate and loss of strain energy is very strong, with no 


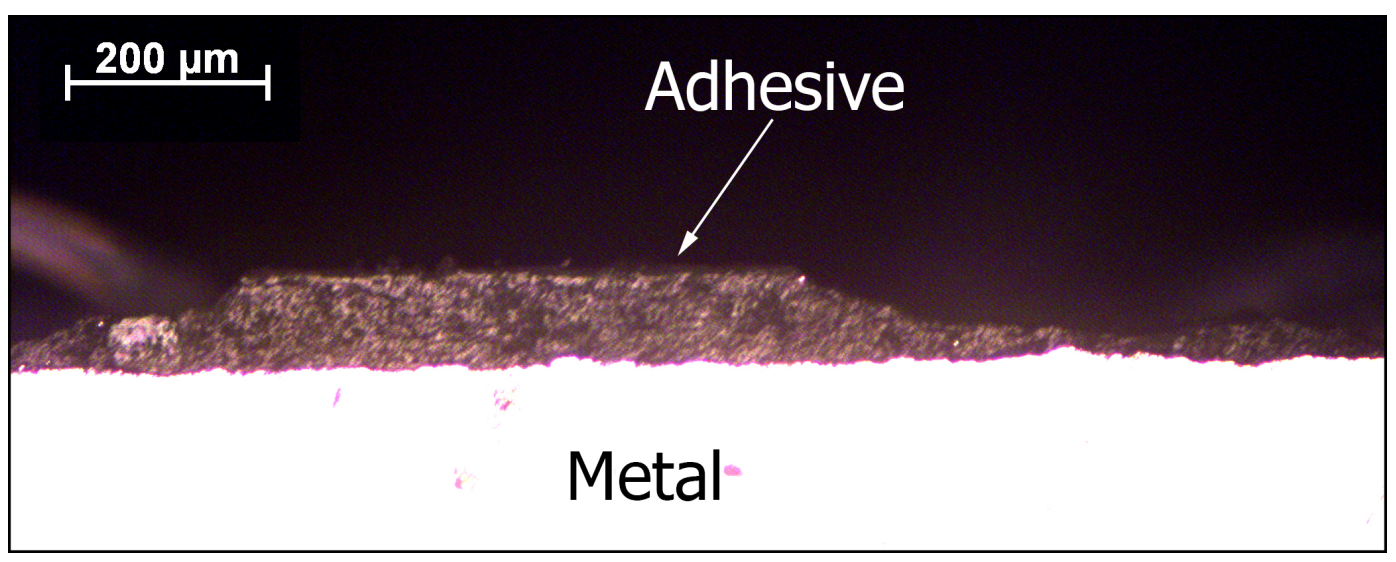

Figure 6: Micrograph of the lower DCB arm of specimen C-001, showing a side view of the crack. Crack growth direction was from left to right.
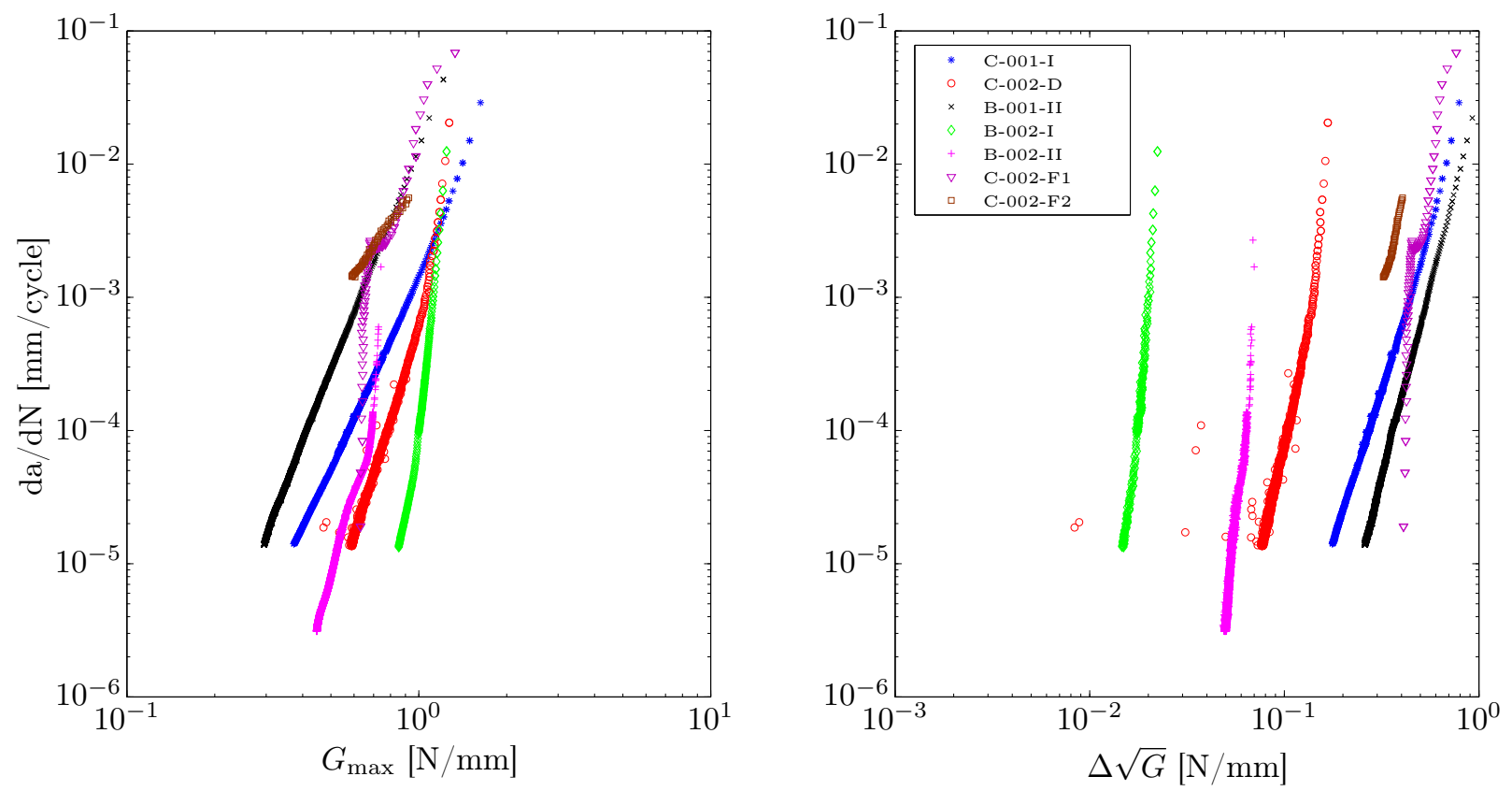

Figure 7: Crack growth rate versus maximum SERR and the SERR range. The data for experiments C-001-I, C-002-D, B-001II, B-002-I and B-002-II were previously presented in [31]. Due to the curve fitting process being further refined for this paper, the curves presented for B-002-I and B-002-II in [31] are slightly different.

apparent R-ratio dependence. A power-law can be fit through both sets of curves, resulting in an equation of the form:

$$
\frac{d A}{d N}=C\left(\frac{d U}{d N}\right)^{n}
$$

For the cyclic energy loss this gives $C=0.8076$ and $n=0.8408\left(R^{2}=0.9869\right)$. For the total energy loss this results in $C=0.7792$ and $n=0.8356\left(R^{2}=0.8704\right)$. The correlation between energy loss and crack growth is better when the loss of cyclic energy is considered, rather than the total loss of energy. The reason for this difference is unclear at present, but it may be related to the inaccuracy in the calculation of the 

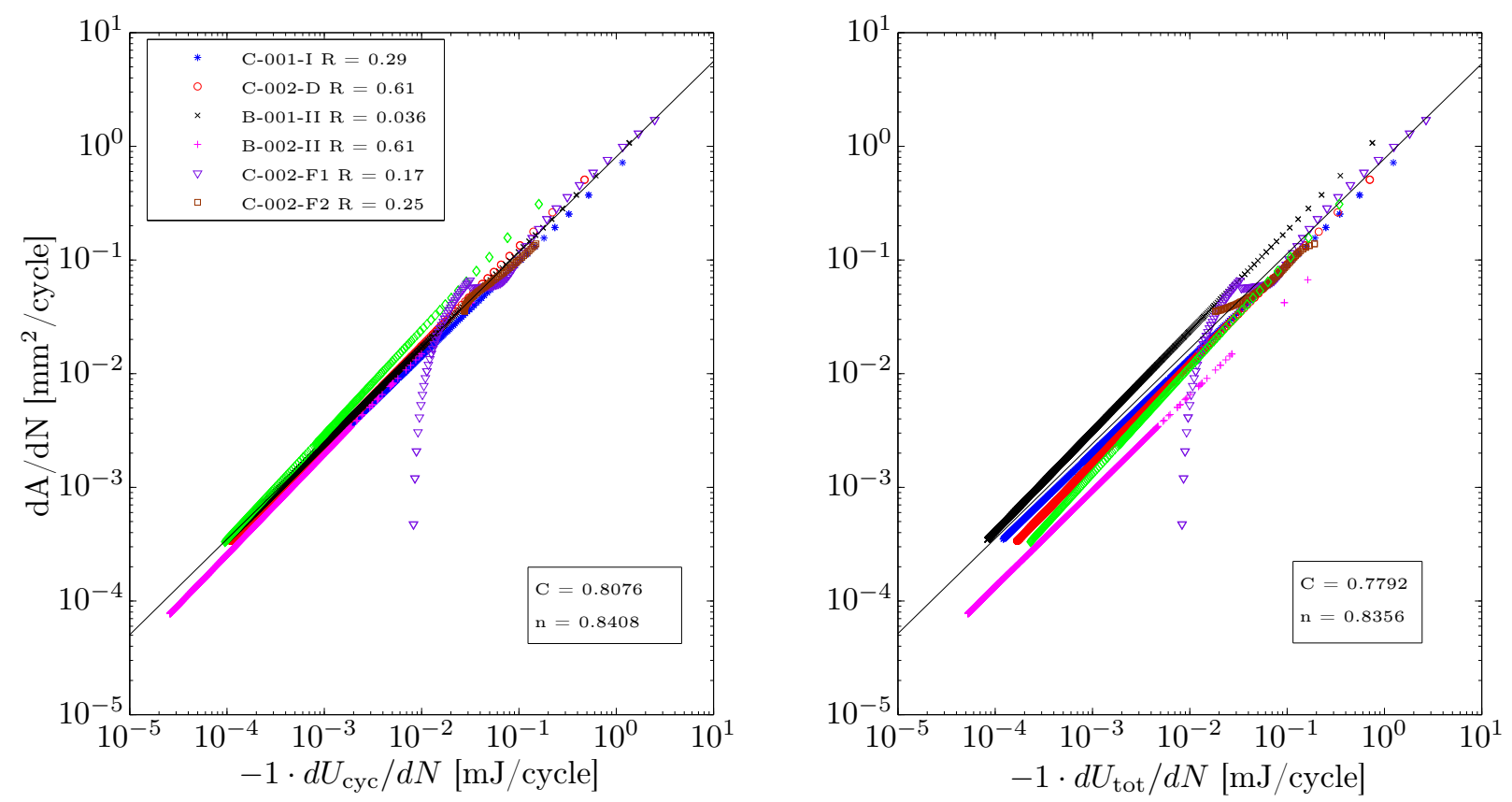

Figure 8: Crack growth rate (in terms of surface area, assuming a straight crack front) versus loss of cyclic energy and loss of total energy. See figure 1 for the definition of $U_{\text {cyc }}$ and $U_{\text {tot }}$.

strain energy discussed in section 4.

\subsection{Constancy of the required energy}

A notable feature of the obtained correlations is that the exponent $n$ is not equal to unity. This implies that the amount of energy required per unit of crack growth is not constant. A possible explanation for this behaviour has been proposed in the work of Broberg [37]. Broberg suggested that there is an "end-zone" where the actual crack growth process takes place, and that this zone is surrounded by a zone in which there is plastic deformation. As the plastic deformation absorbs energy, the amount of energy flowing from the plastic zone to the end-zone is not equal to the amount of energy flowing from the surrounding material to the plastic zone. Rather, only some fraction of the energy supplied to the plastic zone flows on to the end-zone. Broberg suggests that this fraction is not constant, but will in fact decrease as the crack grows, due to an increase in plastic deformation. If one only considers the amount of energy flowing into the plastic zone, the result would be that the apparent amount of energy required for a unit of crack growth is not constant. Although Broberg's hypothesis regarding the size and configuration of the process zone(s) was not investigated in this research, it is certainly clear that the amount of energy released per unit of crack growth is indeed not constant.

Broberg's work dealt with the zone in the vicinity of the crack tip. However the strain energy measured in this research was that contained in the entire specimen. Thus an alternative hypothesis that would be consistent with the presented results is the existence of energy dissipation mechanisms remote from the crack tip. If the fraction of the released strain energy absorbed by these mechanisms would be non-constant that would also give the results seen here. However it is unclear what these mechanisms could be. Upon unloading the specimens returned to their original size and shape, indicating that plastic deformation away from the crack tip was non-existent or very limited in extent. Hence the change in energy required per unit crack growth is most likely related to processes occurring in the immediate vicinity of the crack tip.

As crack length was measured along a straight line, an increase in crack kinking or branching at very small scales could also cause the appearance of greater energy being required for a certain crack extension. 
In this case the actual amount of energy required per unit of extension can remain the same, but due to the actual crack extension length being greater than the apparent straight-line length the amount of energy required per unit of extension will appear to be higher.

\subsection{Applicability to other materials}

The results presented in figure 8 were obtained for crack growth in an epoxy adhesive. However it is expected that the results will hold for more types of materials. In particular, the results should at least hold for crack growth behaviour that is continuous and shows a log-linear dependence on SIF or SERR. Examples are fatigue crack growth in metals or fatigue delamination growth in fibre reinforced polymer composites. Whether the observed correlation also holds for other types of crack growth, such as the alternating arrest and growth seen in certain types of polymers, was not considered.

\subsection{Thickness effect}

Only one adhesive thickness was investigated in this work. It is known that the thickness of the adhesive can effect crack growth, in particular by changing the constaint on the crack, or by changing the fracture mechanism (see e.g. [38, 39]). Although the thickness effect could not be investigated in this research, it is expected that the main finding, i.e. that there exists a correlation between $\mathrm{d} U / \mathrm{d} N$ and $\mathrm{d} a / \mathrm{d} N$, will still be valid. Although the change in constraint may affect the ratio of energy flowing to the crack growth compared to energy flowing to other dissipation mechanisms, and thereby change the parameters $C$ and $n$, the basic principle of the energy balance should still hold. Thus, regardless of the thickness there should be a correlation between $\mathrm{d} U / \mathrm{d} N$ and $\mathrm{d} a / \mathrm{d} N$ that is unaffected by the R-ratio.

\section{Applicability to crack growth predictions}

Although figure 8 shows the fundamental relationship between strain energy loss and crack growth rate, it is of limited use if one desires to create a predictive model. Whereas the SERR can be obtained purely on theoretical grounds, by calculating the loss of stiffness due to an infinitesimal crack extension, the loss of strain energy in a fatigue cycle will depend on the instantaneous value of $G$ at each point in the cycle, as well as the actual crack extension at that point in the cycle. After all, the energy released by crack growth is the product of the SERR and the size of the crack growth increment. In other words the total release of strain energy during a cycle can be written as:

$$
\frac{d U}{d N}=\int G(a) d a
$$

if the limits of integration are chosen suitably to cover the growth of the crack during one cycle. Note that $G$ is not only a function of $a$, but also of $P$ and $d$. However $P$ and $d$ can themselves be seen as a function of $a$ on the scale of one fatigue cycle.

The issue if one wants to perform a crack growth prediction is that equation 13 requires knowledge not only of $G$, but also of $d a / d N$, in order to determine the limits of integration. Thus $d U / d N$ can only be calculated (whether from $G$ or from $P$ and $d$ measurements) a posteriori, after a fatigue test has been performed. For a prediction one obviously needs a value that can be calculated a priori.

To obtain such a value, note that if the loss of strain energy $(d U / d N)$ is divided by the crack growth rate $(d a / d N)$ one obtains $d U / d a$, which is of course an expression of the SERR, here denoted $G^{*}$. As $d U / d N$ and $d a / d N$ have been determined on the scale of one cycle (i.e. crack growth and loss of energy are both assumed to be constant during a single cycle) $G^{*}(N)$ can be understood as the average amount of strain energy released per unit of crack extension during cycle number $N$. If the load cycle and initial crack length are known, this should provide sufficient information to at least approximate $G^{*}$. Further research is now being undertaken to determine how to do this.

The correlation between crack growth rate and $G^{*}$ is shown in figure 9 , which was based on the loss of cyclic energy $\left(d U_{\text {cyc }} / d N\right)$. Note that this figure shows the crack growth rate and strain energy release rate in terms of crack area (assuming a straight crack front), rather than crack length. The solid line is 


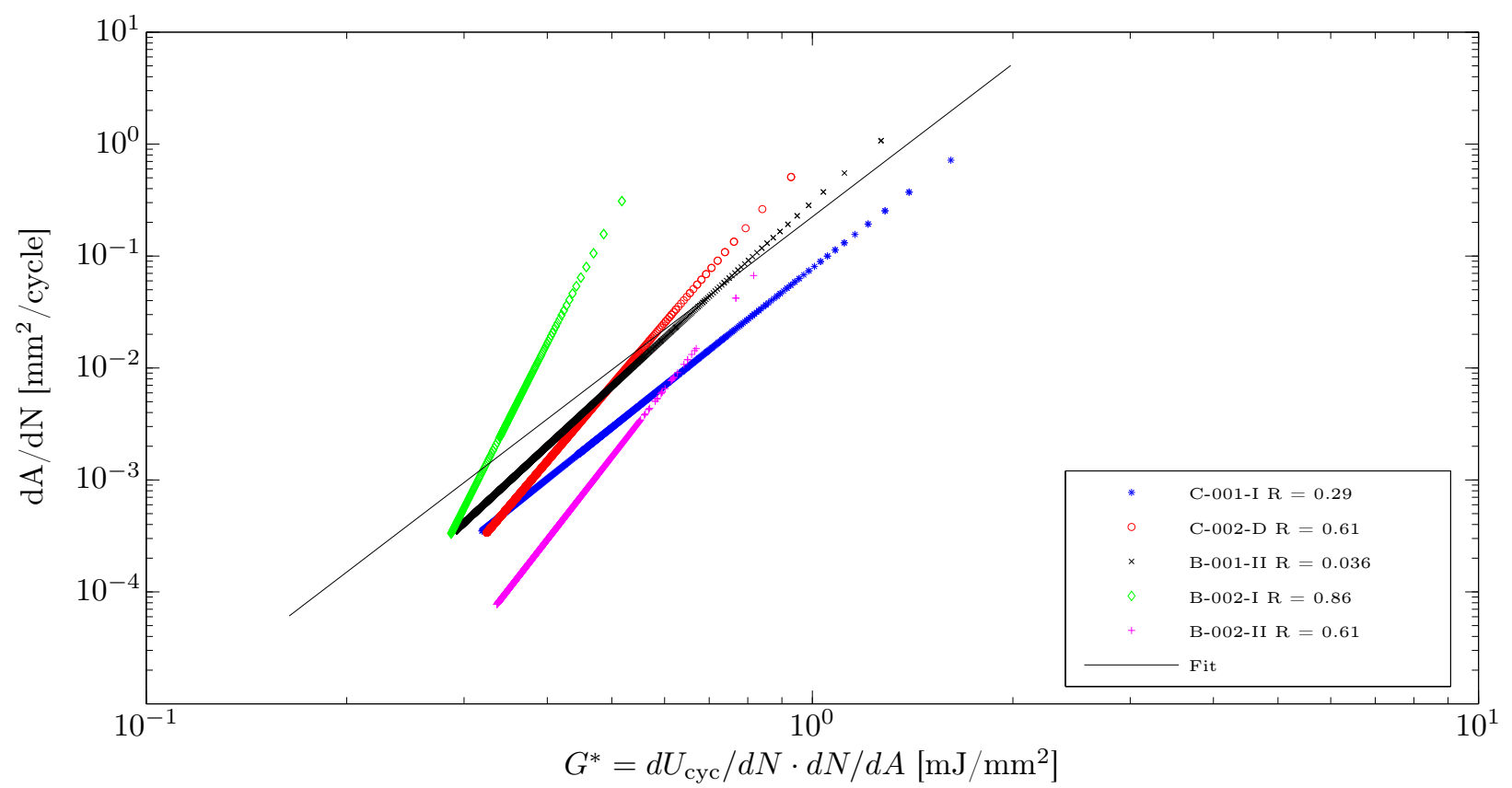

Figure 9: Correlation between the crack growth rate (in terms of new surface area per cycle) and the average strain energy release rate. The fit line is not a fit of the curves in this figure, but was obtained from a curve fit through the collected $d U_{\text {cyc }} / d N$ vs $d A / d N$ curves shown in figure 8 .

not a fit through the $d A / d N$ vs $G^{*}$ curves, but is derived from a fit through the $d A / d N$ vs $d U_{\text {cyc }} / d N$ data shown in figure 8. As the anomalous behaviour of the force controlled experiments (C-002-F1 and C-002-F2) visible in figures 7 and 8 is thought to be an artefact of measurement errors, rather than the actual physical response of the specimen, only the displacement controlled experiments were used to produce figure 9 . The $G^{*}$ versus crack growth rate curves show a greater spread than the energy loss versus crack growth rate curves. This is thought to be caused by the procedure to determine $G^{*}$ (i.e. division by $d A / d N$ ) magnifying small differences in the energy loss per cycle based curves.

Looking at figure 9 there also appears to be an R-ratio dependence, which was not expected. The slope of the curves corresponding to high R-ratios (0.61 and 0.86) is noticeably higher than the slope of the curves corresponding to low R-ratios $(0.29$ and 0.036$)$ and the slope of the fit derived from the collective $d U / d N$ versus $d A / d N$ behaviour. This was thought to be related to the error in the strain energy calculation discussed in section 4.1 .

A correction was applied by estimatng the size of the error using the method described in section 4.1 and adding this value to the calculated loss of strain energy. $G^{*}$ was then recalculated with this corrected value. Figure 10 shows the result.

Compared to figure 9 the curves are closer together, but there still appears to be an R-ratio effect visible in the slope of the curves. As was discussed in section 4, the size of the error in the strain energy release calculation will be R-ratio dependent, and the correction method employed in this work only provides a rough estimation of the magnitude of the error. Furthermore, comparing figure 8 with figures 9 and 10 , it appears that the procedure used to calculate $G^{*}$ magnifies small variations in the slopes of the $d U / d N$ versus $d A / d N$ curves. Thus it is expected that a more accurate calculation of the strain energy will remove the observed R-ratio effect and produce a single relationship between $G^{*}$ and $d A / d N$. 


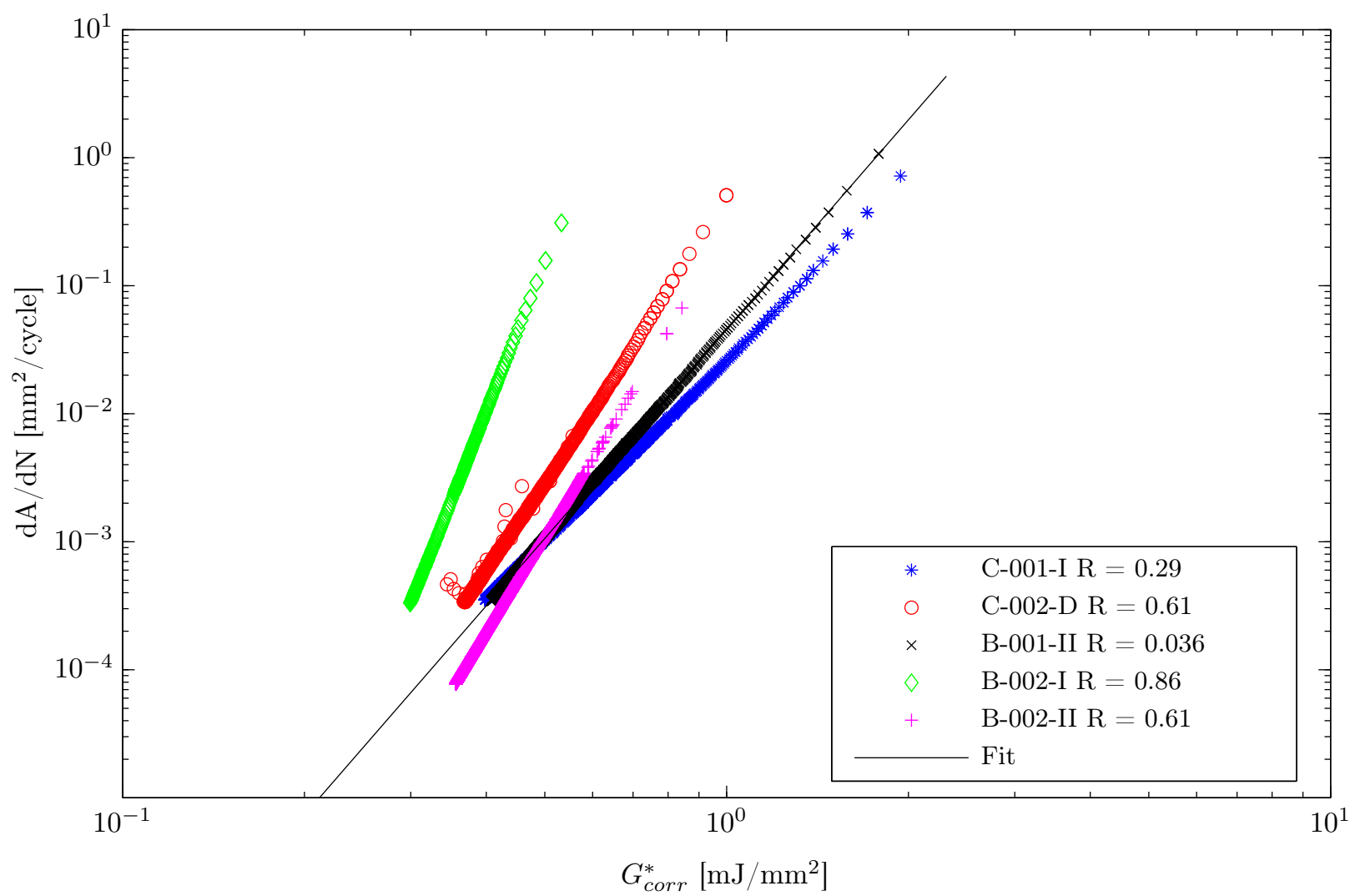

Figure 10: Correlation between crack growth rate and the average strain energy release rate $G^{*}$ including an approximate correction for the underestimation of the released strain energy. The fit line was obtained from a fit through the $d U_{\text {cyc }} / d N$ versus $d A / d N$ curves, including a correction for the underestimation of the released strain energy as described in section 4.1 .

\subsection{Relationship between $G^{*}$ and stress intensity factor}

By making use of Irwin's relationship (equation 2) any SERR value can in principle be related to a SIF value. Thus $G_{\max }$ can be related to $K_{\max }$ and $\Delta \sqrt{G}$ can be related to $\Delta K$. As $K$ describes a stress field, and thus also a strain field, it can be used to describe the stress and deformation state around the crack tip. Thus Irwin's relationship can be used to relate $G$ to the stress and strain fields around the crack tip. In principle Irwin's relationship can also be used to equate $G^{*}$ to some $K$ value, however the physical meaning of this is not clear. $G^{*}$ represents an average $G$ value and thus the corresponding $K^{*}$ value would also represent some kind of average stress field around the crack tip. Since it is currently still being investigated how to calculate $G^{*}$ from a given loading cycle, and thus how $G^{*}$ relates to the entire $G$ cycle, it is also unclear what the physical relevance of $K^{*}$ is. Once the relationship between $G^{*}$ and the $G$ cycle has been established, this will hopefully also shed light on the connection between $G^{*}$ and the stresses and deformations around the crack tip.

\section{Conclusions and future work}

This paper has highlighted the shortcomings of the current approach of correlating fatigue disbond growth to the strain energy release rate. It was pointed out that the SERR is an instantaneous value and by itself does not provide information on the actual amount of energy released, whereas the energy balance concept of Griffith demands the equality of the actual energy release and consumption over the entire cycle. The fact 
that the various fatigue test data can (essentially) be collapsed onto a single $\mathrm{d} a / \mathrm{d} N$ versus $\mathrm{d} U$ cyc $/ \mathrm{d} N$ curve suggests that the use of $G_{\max }$ or $\Delta G$ alone cannot provide sufficient information to uniquely characterise the disbond growth rate. Furthermore this suggests that the so-called R-ratio effect is merely the consequence of this lack of a unique description of the load cycle.

If the actual release of strain energy in a cycle is measured it is found to be very strongly correlated to the crack growth rate. This relationship does not depend on the R-ratio or mean stress. Although the release of strain energy can not be predicted a priori, the relationship between strain energy loss and crack growth rate can be used as the basis for a predictive model. By dividing the loss of energy by the crack growth rate one obtains a relationship between the average strain energy release per unit crack growth (called here $G^{*}$ ) and the crack growth rate. In the present work this did not produce one unique relationship between $G^{*}$ and the crack growth rate. This is thought to be caused by the underestimation of the strain energy release as a result of assuming linearity of the force-displacement curve. Future work will examine ways of refining the experimental technique and data analysis to account for this error. A more accurate measurement of the force-displacement curve, allowing the calculation of the strain energy using more points than just $\left(P_{\max }, d_{\max }\right)$ and $\left(P_{\min }, d_{\min }\right)$ is expected to give better results. In addition, research will focus on how to determine $G^{*}$ for a given load cycle. This will likely require a better understanding of which parts of the load cycle contribute to crack growth and in what way.

Another question that remains unanswered is whether the SERR should indeed be seen as the driving force for fatigue crack growth. The release of strain energy, as proposed by Griffith, is a consequence of crack growth resulting in a removal of internal constraints. Although the correlation between crack growth and strain energy release is very strong, this does not mean that the strain energy release is indeed the driving force for crack growth. What then is the driving force remains for the moment an open question.

\section{Acknowledgement}

This work was made possible by the Netherlands Organisation for Scientific Research (NWO) by a grant from the Mosaic program under project number 017.009.005.

\section{References}

[1] J. A. Pascoe, R. C. Alderliesten, R. Benedictus, Methods for the prediction of fatigue delamination growth in composites and adhesive bonds - a critical review, Engineering Fracture Mechanics 112-113 (2013) 72-96.

[2] A. A. Griffith, The phenomena of rupture and flow in solids, Philosophical Transactions of the Royal Society of London Series A, Containing Papers of a Mathematical or Physical Character 221 (1921) 163-198.

[3] E. Orowan, Fracture and strength of solids, Reports on Progress in Physics 12 (1) (1949) 185.

[4] E. Orowan, Fundamentals of brittle behavior in metals, in: W. Murray (Ed.), Fatigue and Fracture of Metals, Massachusetts Institute of Technology and John Wiley \& Sons, New York, 1952, pp. 139-167.

[5] G. R. Irwin, Fracture dynamics, in: G. Sachs (Ed.), Fracturing of Metals, American Society for Metals, Cleveland, 1948.

[6] G. R. Irwin, J. Kies, Fracturing and fracture dynamics, Welding Journal - Research Supplement 31 (Feb) (1952) 95s-100s.

[7] G. R. Irwin, J. Kies, Critical energy rate analysis of fracture strength, Welding Journal - Research Supplement 33 (Apr) (1954) 193s-198s

[8] G. R. Irwin, Analysis of stresses and strains near the end of a crack traversing a plate, ASME Journal of Applied Mechanics 24 (1957) 361-364.

[9] H. Westergaard, Bearing pressures and cracks, Journal of Applied Mechanics 61 (01) (1939) A49-A53.

[10] D. Miannay, Fracture Mechanics, Mechanical Engineering Series, Springer, New York, 1998.

[11] N. Perez, Fracture Mechanics, Springer US, Boston, 2004.

[12] P. Paris, M. Gomez, W. Anderson, A rational analytic theory of fatigue, The Trend in Engineering 13 (1961) 9-14.

[13] P. Paris, The fracture mechanics approach to fatigue, in: 10th Sagamore Army Materials Research Conference, Syracuse University Press, 1964, pp. 107-132.

[14] P. Paris, F. Erdogan, A critical analysis of crack propagation laws, Journal of Basic Engineering 85 (4) (1963) $528-533$.

[15] G. Roderick, R. Everett, J. Crews Jr, Debond propagation in composite reinforced metals, Tech. Rep. NASA TM X-71948, NASA (1974).

[16] S. Mostovoy, E. Ripling, Flaw Tolerance of a Number of Commercial and Experimental Adhesives, Polymer Science and Technology 9B, Plenum Press, New York, 1975, pp. 513-562.

[17] A. Poursartip, N. Chinatambi, Fatigue damage development in notched $\left(0_{2} / \pm 45\right)_{s}$ laminates, in: P. A. Lagace (Ed.), Composite Materials: Fatigue and Fracture, Second Volume, ASTM STP 1012, American Society for Testing and Materials, Philadelphia, 1989, pp. 45-65. 
[18] J. Andersons, M. Hojo, S. Ochiai, Model of delamination propagation in brittle-matrix composites under cyclic loading, Journal of Reinforced Plastics and Composites 20 (5) (2001) 431-450.

[19] G. Allegri, M. I. Jones, M. R. Wisnom, S. R. Hallett, A new semi-empirical model for stress ratio effect on mode II fatigue delamination growth, Composites Part A 42 (7) (2011) 733-740.

[20] G. Allegri, M. R. Wisnom, A non-linear damage evolution model for mode II fatigue delamination onset and growth, International Journal of Fatigue 43 (0) (2012) 226-234.

[21] G. Allegri, M. R. Wisnom, S. R. Hallett, A new semi-empirical law for variable stress-ratio and mixed-mode fatigue delamination growth, Composites Part A: Applied Science and Manufacturing 48 (0) (2013) 192-200.

[22] M. Hojo, K. Tanaka, C. G. Gustafson, R. Hayashi, Effect of stress ratio on near-threshold propagation of delimination fatigue cracks in unidirectional CFRP, Composites Science and Technology 29 (4) (1987) 273-292.

[23] M. Hojo, S. Ochiai, C.-G. Gustafson, K. Tanaka, Effect of matrix resin on delamination fatigue crack growth in CFRP laminates, Engineering Fracture Mechanics 49 (1) (1994) 35-47.

[24] J. Andersons, M. Hojo, S. Ochiai, Empirical model for stress ratio effect on fatigue delamination growth rate in composite laminates, International Journal of Fatigue 26 (6) (2004) 597-604.

[25] D. R. Atodaria, S. K. Putatunda, P. K. Mallick, A fatigue crack growth model for random fiber composites, Journal of Composite Materials 31 (18) (1997) 1838-1855.

[26] D. R. Atodaria, S. K. Putatunda, P. K. Mallick, Delamination growth behavior of a fabric reinforced laminated composite under mode I fatigue, Journal of Engineering Materials and Technology 121 (3) (1999) 381-385.

[27] D. R. Atodaria, S. K. Putatunda, P. K. Mallick, Fatigue crack growth model and mechanism of a random fiber SMC composite, Polymer Composites 20 (2) (1999) 240-249.

[28] R. Jones, S. Pitt, A. J. Bunner, D. Hui, Application of the Hartman-Schijve equation to represent mode I and mode II fatigue delamination growth in composites, Composite Structures 94 (4) (2012) 1343-1351.

[29] R. Jones, S. Stelzer, A. J. Brunner, Mode I, II and mixed mode I/II delamination growth in composites, Composite Structures 110 (2014) 317-324.

[30] R. Khan, Mode I fatigue delamination growth in composites, Phd thesis, Delft University of Technology (2013).

[31] J. A. Pascoe, R. C. Alderliesten, R. Benedictus, Towards understanding fatigue disbond growth via cyclic strain energy $20^{\text {th }}$ European conference on fracture, Procedia Materials Science 3 (ECF-20) (2014) 610-615.

[32] J. A. Pascoe, R. C. Alderliesten, R. Benedictus, Damage tolerance of adhesive bonds - dataset I, available at the 3TU data centre via: http://dx.doi.org/10.4121/uuid:a2997418-682b-46f5-a988-ba0c88d7350e (2013).

[33] ASTM, ASTM Standard D5528-01: Standard test method for mode I interlaminar fracture toughness of unidirectional fiber-reinforced polymer matrix composites, ASTM International, West Conshohocken, PA, 2007.

[34] J. A. Pascoe, R. C. Alderliesten, R. Benedictus, Damage tolerance of adhesive bonds - dataset Ia, available at the 3TU data centre via: http://dx.doi.org/10.4121/uuid:e42844b3-e9f8-420a-b896-d35106530793 (2014).

[35] D. Bürger, C. D. Rans, R. Benedictus, Influence of fabric carrier on the fatigue disbond behavior of metal-to-metal bonded interfaces, The Journal of Adhesion 90 (5-6) (2013) 482-495.

[36] C. Rans, R. C. Alderliesten, R. Benedictus, Misinterpreting the results: How similitude can improve our understanding of fatigue delamination growth, Composites Science and Technology 71 (2011) 230-238.

[37] K. B. Broberg, On stable crack growth, Journal of the Mechanics and Physics of Solids 23 (3) (1975) $215-237$.

[38] M. Imanaka, T. Iwata, Effect of adhesive layer thickness on fatigue strength of adhesively bonded butt, scarf and butterfly type butt joints, Int J Fract 80 (4) (1996) R69-R76.

[39] K. Kageyama, I. Kimpara, T. Suzuki, I. Ohsawa, K. Esaki, Effect of interleaf thickness of mode II fatigue delamination growth of interleaved carbon/epoxy, Key Engineering Materials 137 (1998) 187-194. 